\title{
The role of Nanocartography in the Development of Automated TEM
}

Matthew Olszta ${ }^{1}$, Kevin Fiedler ${ }^{2}$, Steven Spurgeon ${ }^{3}$, Sarah Reehl ${ }^{3}$ and Derek Hopkins ${ }^{3}$

${ }^{1}$ Pacific Northwest National Laboratory, Washington, United States, ${ }^{2}$ United States, ${ }^{3}$ Pacific Northwest National Laboratory, United States

The development of automated transmission and scanning transmission electron microscopy (S/TEM) for materials science analysis has been a long sought-after goal. Following the lead of the biological microscopy community, which has already vested considerable resources and research interest into automating sample analysis, the materials community is now recognizing a similar need. With the advent of next generation machine learning (ML) and artificial intelligence (AI) algorithms to tackle more complex problems and automatically decipher intricate pattern recognition, there will be a diminished need for user interaction. This does not preclude expert user interaction, but the more integrated intelligent learning is woven into the operation of the electron microscope the more optimized and repeatable microscopy can become transformed. There are two major thrusts towards proper integration of automation into the current workflow, the first being ML/AI that can assist in adequately tackling large data sets and complex pattern recognition, and the second is the ability to accurately control the stage through predictive tilting to accommodate travel both through the crystallography of a sample as well along physical interfaces and microstructures. Nanocartography was developed as a means to accomplish the second goal [1].

Nanocartography combines predictive stage tilting and crystallography with advanced documentation to perform highly repeatable and accurate measurements (Figure 1). Within the electron microscopy literature there are a number of publications that have individually tackled these issues [2-6], but most often from the standpoint of knowledge of existing crystal systems. This approach of a priori analysis (i.e., having roadmaps of specific candidate crystals) precludes the ability to solve unknowns, and as well does not address tilt control of physical objects such as grain boundaries and interfaces. Nanocartography approaches the system as a whole, irrespective of specific crystal, allowing for microscopists to eventually build their own sample analysis methodologies. More importantly, it lends itself to more rigorous, detailed, and meticulous reporting of science and decision making. Approaching sample analysis in this manner provides a blueprint for automation in any electron microscope.

The control of double tilt stage motion was integral to the initial stages of nanocartography [1]. As will be demonstrated through the use of microscope interface programs such as PyJEM, the ability to digitally control the stage and probe motion are imperative to designing next generation experiments. We will discuss how off hours analysis through automated montaging and oblique tilt series can be accomplished with minimal input from the user. Coupled with signal integration from multiple detectors, this allows for multimodal approaches in understanding more about each sample in less time. Combining nanocartography and ML/AI image analysis will provide an intelligent future for automated analysis programs such as AutoEM. 

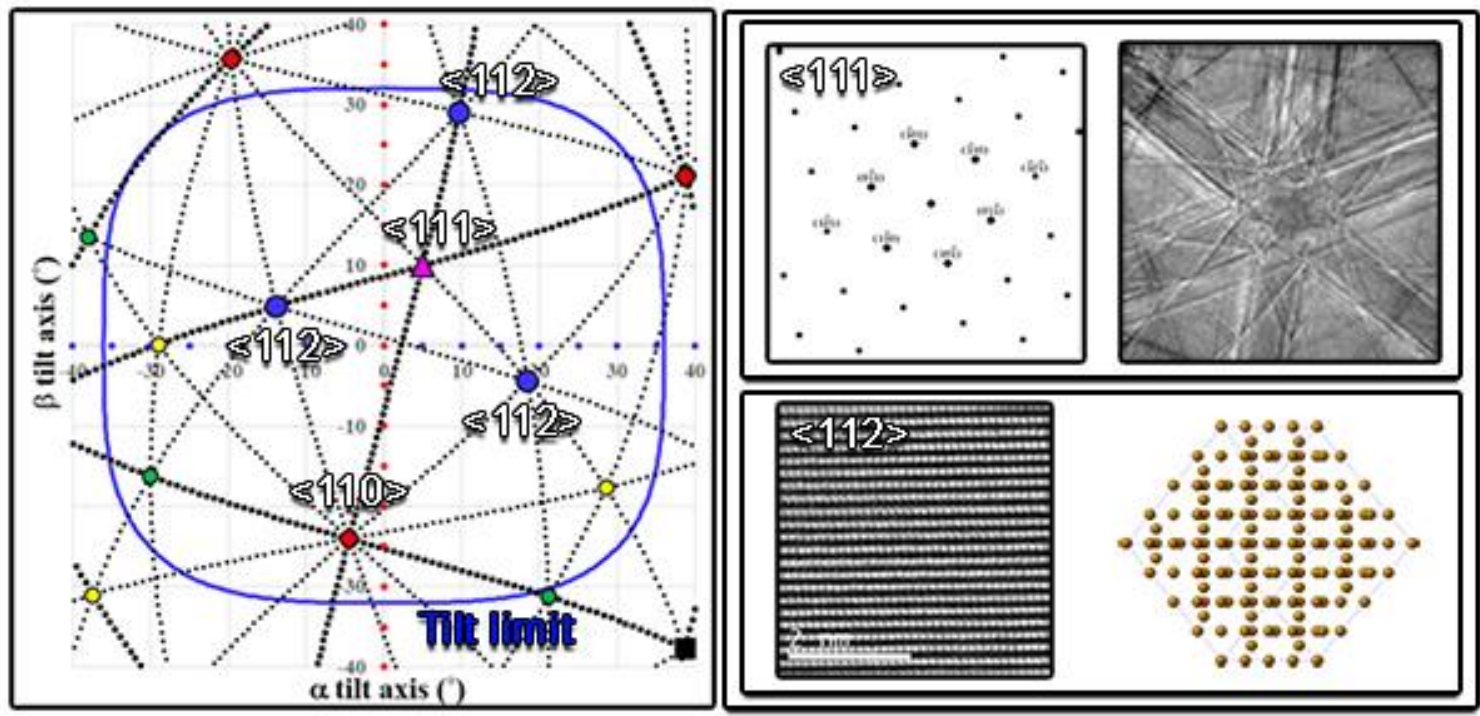

Figure 1.

Figure 1. Nanocartography utilizes predictive stage tilting and crystallography to guide the user in performing high end analytical electron microscopy.

References

1. Olszta, M. and K. Fiedler, Nanocartography in the Age of Automated TEM. Microscopy and Microanalysis, 2020. 26(S2): p. 32-32.

2. Cautaerts, N., R. Delville, and D. Schryvers, ALPHABETA: a dedicated open-source tool for calculating TEM stage tilt angles. Journal of Microscopy, 2018. 273.

3. Duden, T., et al., K-space Navigation for Accurate High-angle Tilting and Control of the TEAM Sample Stage. Microscopy and Microanalysis, 2009. 15(S2): p. 1228-1229.

4. $\quad$ Li, X.-Z., SPICA: stereographic projection for interactive crystallographic analysis. Journal of Applied Crystallography, 2016. 49(5): p. 1818-1826.

5. Liu, Q., A simple and rapid method for determining orientations and misorientations of crystalline specimens in TEM. Ultramicroscopy, 1995. 60(1): p. 81-89.

6. Xie, R.-X. and W.-Z. Zhang, [tau]ompas: a free and integrated tool for online crystallographic analysis in transmission electron microscopy. Journal of Applied Crystallography, 2020. 53(2). 